\title{
Ocupação diferencial do habitat por vespas do gênero Trypoxylon (Trypargilum) Latreille (Hymenoptera, Crabronidae)
}

\author{
Mariana Marchi Santoni ${ }^{1}$, Antonio Domingos Brescovit ${ }^{2} \&$ Marco Antonio Del Lama $^{1}$
}

${ }^{1}$ Laboratório de Genética Evolutiva de Himenópteros, Departamento de Genética e Evolução, Universidade Federal de São Carlos. Rodovia

Washington Luis (SP-310), km 235, 13565.905 São Carlos-São Paulo, Brasil.mmsantoni@gmail.com; dmdl@ufscar.br

${ }^{2}$ Laboratório de Artrópodes, Instituto Butantan, Av. Vital Brasil, 1500, Butantã, 05503.900 São Paulo-SP, Brasil. anyphaenidae@butantan.gov.br

\begin{abstract}
Differential occupation of the habitat by Trypoxylon (Trypargilum) Latreille wasps (Hymenoptera, Crabronidae). Wasps of the genus Trypoxylon are solitary and females mass-provision their nests with paralyzed spiders. Some species use successfully trap-nests for nesting foundation, making easier their sampling and study. This paper reports data about nesting biology of four species of Trypoxylon (Trypargilum) - T. rogenhoferi, T. lactitarse, T. aurifrons and T. nitidum. These species were sampled by trap-nests during three years in Araras and two years in São Carlos and Rifaina (São Paulo). These areas were subdivided into sampling sites. A total of 2,698 nests of solitary hymenopterans were collected and most of them $(2,268)$ were founded by Trypoxylon species. Nesting activity was higher in the warm, rainy season (October-March). The trap-nests used by the different species showed significantly different dimensions. Araneidae was the spider family mainly used for provision, but a species-specific provisioning was observed. The most important parasitoid of the four species was Melittobia, but adult chrysidids, ichneumonids, chalcidids and sarcophagids also attacked their nests. In the three areas, the different species of Trypoxylon coexist temporally but each of them built their nests frequently in a specific site. This result may suggest differential occupation of the habitat. This habitat partition of an apparently homogeneous area may be a result of an "apparent competition" shaped by shared natural enemies.
\end{abstract}

KEYWORDS. Apparent competition; Araneidae; habitat partition; parasitoid; trap-nest.

RESUMO. Ocupação diferencial do habitat por vespas do gênero Trypoxylon (Trypargilum) Latreille (Hymenoptera, Crabronidae). Vespas do gênero Trypoxylon apresentam comportamento solitário e aprovisionam seus ninhos de forma massiva com aranhas paralisadas. Algumas espécies utilizam cavidades preexistentes para nidificação, o que facilita sua amostragem e estudo. Neste trabalho, dados sobre a biologia de nidificação de quatro espécies de Trypoxylon (Trypargilum) - T. rogenhoferi, T. lactitarse, T. aurifrons e T. nitidum - são apresentados. As espécies foram amostradas por meio de ninhos-armadilha durante três anos em Araras e dois anos em Rifaina e São Carlos (São Paulo). As localidades de estudo foram subdivididas em sítios de amostragem. Foram obtidos 2.698 ninhos de himenópteros solitários, dos quais 2.268 foram fundados por espécies de Trypoxylon. Intensa atividade de nidificação foi observada principalmente na estação quente e chuvosa (outubro-março). Os ninhos-armadilha utilizados para nidificação pelas diferentes espécies apresentaram dimensões significativamente diferentes. A família de aranhas mais utilizada para aprovisionamento foi Araneidae; no entanto, as espécies de vespas diferiram quanto às espécies forrageadas. O principal parasitóide das quatro espécies foi Melittobia, mas indivíduos das famílias Chrysididae, Ichneumonidae, Chalcididae e Sarcophagidae também foram verificados. Observou-se que as espécies de Trypoxylon coexistem temporalmente e que nas três localidades cada espécie nidificou com maior freqüência em um sítio particular, sugerindo uma ocupação diferencial do habitat. A partição do habitat aparentemente homogêneo pode ser resultado de "competição aparente" mediada por inimigos naturais comuns.

PALAVRAS-CHAVE. Araneidae; competição aparente; ninho-armadilha; parasitóide; partição de habitat.

A Ordem Hymenoptera agrupa espécies de abelhas, vespas e formigas que apresentam diferentes níveis de organização social. Cerca de $90 \%$ das espécies de vespas apresentam comportamento solitário, caracterizado pela independência das fêmeas na construção e aprovisionamento das células de cria (O’Neill 2001). Algumas espécies constróem seus ninhos em cavidades preexistentes, como os representantes das famílias Vespidae, Pompilidae, Sphecidae e Crabronidae (Gauld \& Bolton 1996).

Trypoxylon Latreille, 1796 é o gênero mais diverso dentro da família Crabronidae. Apresenta ampla distribuição geográfica, mas sua maior diversidade está na região Neotropical. São vespas solitárias e a fêmea exibe cuidado maternal na forma de construção e aprovisionamento dos ninhos, onde os imaturos se desenvolvem até o estágio adulto (Coville 1982).

O subgênero Trypargilum Richards, 1934, restrito ao Novo Mundo (Hanson \& Menke 1996), é constituído de vespas solitárias cujas fêmeas aprovisionam seus ninhos com aranhas paralisadas. Neste grupo, os machos apresentam o comportamento de macho-guarda, permanecendo junto ao ninho durante sua construção.

Estas vespas usualmente nidificam com sucesso em cavidades artificiais (Coville 1982) e a utilização de ninhosarmadilha facilita sua amostragem, produzindo dados sobre abundância, riqueza e sazonalidade (Camillo et al. 1995; Loyola \& Martins 2006; Morato \& Martins 2006), arquitetura intranidal, utilização de presas e inimigos naturais (Buschini et al. 2006; 
Buschini \& Wolff 2006; Camillo 1999; Camillo et al. 1993, 1994, Camillo \& Brescovit 1999; Camillo \& Brescovit 2000; Coville \& Coville 1980; Garcia \& Adis 1995; Santoni \& Del Lama 2007), comportamento do macho-guarda (Coville \& Coville 1980; Brockmann \& Grafen 1992) e investimento parental (Peruquetti $\&$ Del Lama 2003).

Este trabalho descreve dados relativos à biologia de nidificação de vespas do subgênero Trypargilum em ninhosarmadilha dispostos em três localidades do estado de São Paulo. Aspectos como diversidade, abundância sazonal, estrutura de ninhos, presas utilizadas para aprovisionamento, mortalidade e parasitismo são relatados. Uma possível razão para a utilização diferencial dos sítios de nidificação pelas espécies é discutida.

\section{MATERIALE MÉTODOS}

Áreas de Estudo. Este estudo foi conduzido em três áreas localizadas no estado de São Paulo: o campus de Araras da Universidade Federal de São Carlos (UFSCar) $\left(22^{\circ} 18^{\prime}\right.$ S, $\left.47^{\circ} 22^{\prime} \mathrm{W}, 629 \mathrm{~m}\right)$, o campus da UFSCar em São Carlos ( $22^{\circ} 01^{\prime} \mathrm{S}$, $47^{\circ} 53^{\prime} \mathrm{W}, 850 \mathrm{~m}$ ) e a Fazenda Rio Branco, localizada no município de Rifaina (200.' S, $47^{\circ} 25^{\prime} \mathrm{W}, 575 \mathrm{~m}$ ). Estas áreas apresentam clima Cwa (sistema de Köppen), caracterizado por duas estações bem definidas: uma quente e chuvosa (outubromarço), com pluviosidade elevada e temperaturas acima de $22^{\circ} \mathrm{C}$ e outra fria e seca (abril-setembro), com baixa precipitação e temperatura abaixo de $18^{\circ} \mathrm{C}$.

As coletas em Araras ocorreram entre dezembro de 2003 a março de 2007 em sete sítios; em São Carlos, entre novembro de 2004 a novembro de 2006 em três sítios e em Rifaina, de julho de 2004 a março de 2007 em seis sítios. Em Araras, todos os sítios estavam localizados no interior de construções antigas, próximos a corpos d'água, com vegetação de entorno muito modificada (cana-de-açúcar e/ou pastagem, com alguns traços de vegetação natural de cerrado). Em São Carlos, os sítios de nidificação localizavam-se no interior de construções do campus situadas em área de vegetação introduzida (pastagem) ou típica (cerrado). Em Rifaina, os sítios estavam localizados em construções antigas próximas a uma vegetação natural que está sendo alterada pela introdução da cana-deaçúcar. No entanto, dentro de cada área de nidificação (Araras, São Carlos e Rifaina), os sítios escolhidos apresentavam aparente homogeneidade de condições ambientais como temperatura, umidade e luminosidade. Estes sítios estavam distantes entre si de 70 a 380 m em Araras, de 30 a 101 m em São Carlos e de 42 a $244 \mathrm{~m}$ em Rifaina. No período, foram realizadas 32 coletas em Araras, 14 em São Carlos e 19 em Rifaina.

Os dados meteorológicos do período foram obtidos nos sites da UFSCar campus Araras (www.ufscar.br), do Centro de Pesquisa de Pecuária do Sudeste da EMBRAPA (www.cppse.embrapa.br) e do Centro de Pesquisas Meteorológicas e Climáticas Aplicadas à Agricultura da UNICAMP (orion.cpa.unicamp.br).

Amostragem. As espécies foram amostradas utilizando ninhos-armadilha construídos de bambus secos seccionados a cada nó. Estes bambus apresentavam comprimento ( 8 a 62 $\mathrm{cm})$ e diâmetro interno ( 3 a $20 \mathrm{~mm}$ ) variados, eram agrupados em pacotes de 8 a 12 tubos de dimensões similares e horizontalmente dispostos nos sítios das áreas de estudo. Tomou-se o cuidado de dispor quantidades semelhantes de ninhos-armadilha de variadas dimensões em cada sítio. A cada coleta, foram oferecidos cerca de 1.000 ninhos-armadilha em Araras, 300 em São Carlos e 500 em Rifaina, os quais foram inspecionados a cada 35 dias, aproximadamente.

Todos os tubos utilizados pelas vespas foram substituídos por novos e transferidos para o Laboratório de Genética Evolutiva de Himenópteros (LGEH), onde foram abertos. A maioria dos ninhos que se encontrava em fase de ovo e/ou larva era novamente fechada até que o estágio de pupa fosse atingido. As pupas foram individualmente dispostas em frascos de vidro, identificados com número e posição no ninho e mantidos em local isolado, à temperatura ambiente, até a emergência dos adultos, momento em que a espécie era identificada.

Dados coletados. Todos os ninhos-armadilha utilizados pelas espécies de Trypoxylon tiveram comprimento e diâmetro aferidos. Cada ninho foi tratado como dado independente, já que a construção dos ninhos não foi monitorada. Características da arquitetura intranidal, tais como paredes de fechamento e de fundo, e presença de parasitóides foram anotadas. Estes parasitóides foram identificados após sua emergência. As aranhas utilizadas para o aprovisionamento foram retiradas de ninhos com células de cria em estágio de ovo e armazenadas em álcool 70\% para posterior identificação por especialista. Todas as aranhas coletadas foram depositadas na coleção do Instituto Butantan (Curador: A. D. Brescovit).

Análises estatísticas. As análises estatísticas foram realizadas de acordo com Zar (1999), considerando nível de significância de 5\%, utilizando o programa BioEstat 4.0 (Ayres et al. 2005). Média ( $\overline{\mathrm{x}})$ e Desvio Padrão ( \pm ) são apresentados sempre que necessário. Os valores de comprimento e diâmetro dos ninhos-armadilha utilizados pelas fêmeas das espécies de Trypoxylon das três localidades foram agrupados. O número de células foi estimado considerando somente os ninhos completamente fechados (parede de fechamento).

A correlação de Pearson foi utilizada para verificar associação entre número de ninhos e número total de células em relação às condições sazonais (temperatura média mensal e pluviosidade total referentes a cada período). Análises de Variância (ANOVA) e teste de Mann-Whitney foram utilizados para comparar os comprimentos e diâmetros dos ninhosarmadilha utilizados por cada espécie. Teste de $\chi^{2}$ foi utilizado para comparar taxas de mortalidade e parasitismo em cada espécie em cada localidade e para diferentes espécies na mesma localidade.

\section{RESULTADOS}

Riqueza e Abundância. Foram fundados 2.698 ninhos por himenópteros solitários nas três áreas, sendo $96 \%$ por espécies de vespas das famílias Crabronidae, Sphecidae e Vespidae e 
4\% por espécies de abelhas das famílias Megachilidae e Apidae. Foram coletados 1.403 ninhos em Araras, 241 em São Carlos e 1.054 ninhos em Rifaina. As taxas de ocupação dos tubos (ninhos fundados em relação aos ninhos oferecidos) nas três áreas foram $5 \%, 6 \%$ e $12 \%$, respectivamente.

Espécies de Trypoxylon (Trypargilum) produziram 2.268 ninhos, ou seja, cerca de $84 \%$ dos ninhos amostrados. Foi verificada emergência de adultos em 1.322 ninhos (58\%) fundados por cinco espécies de Trypargilum do grupo nitidum (Coville, 1982): Trypoxylon rogenhoferi Kohl, 1884, T. lactitarse Saussure 1867, T. aurifrons Shuckard, 1837, T. nitidum Smith. 1856 e uma espécie não identificada, Trypoxylon sp. Os outros ninhos ou não apresentaram emergência (31\%) ou os adultos já haviam emergido no momento da coleta no campo (11\%).

Ninhos de Trypoxylon rogenhoferi e T. aurifrons foram coletados nas três áreas, T. nitidum em Araras e São Carlos, e T. lactitarse e Trypoxylon sp. foram encontrados apenas em Araras. Esta localidade apresentou o maior número de espécies de Trypoxylon e Rifaina, maior abundância de ninhos (Tab. I).

Dado o baixo número de ninhos fundados por Trypoxylon sp, não foi possível obter dados consistentes sobre a biologia da nidificação nesta espécie.

Distribuição sazonal e espacial. As espécies de Trypoxylon nidificaram durante todo o período de amostragem, mas com maior frequiência na estação quente e chuvosa nas três localidades (Fig. 1). O número de ninhos de T. rogenhoferi, T. lactitarse, T. aurifrons e T. nitidum apresentou correlação significativa com a temperatura média mensal (Tab. II).

Em Araras, ao longo de três anos, foi possível observar uma diminuição do número de ninhos fundados por cada uma das espécies estudadas (Fig. 1a). Quarenta e cinco por cento dos ninhos foram coletados entre dezembro/2003 a novembro/ 2004, 36\% entre dezembro/2004 a novembro/2005 e 19\% entre dezembro/2005 a março/2007. Em São Carlos e Rifaina, também foi observada uma diminuição do número de ninhos fundados ao longo do período (Fig. $1 \mathrm{~b}$ e 1c, respectivamente).

Foi observada uma ocupação preferencial dos sítios, pois cada espécie nidificou com maior freqüência em um sítio particular. Este comportamento foi observado nas três localidades (Fig. 2). Nestas, a predominância de uma espécie em cada sítio foi verificada desde as primeiras coletas. Em Araras, os sítios 1 e 4 foram ocupados quase exclusivamente por T. lactitarse e T. rogenhoferi, respectivamente, enquanto que os sítios 2 e 3 foram ocupados por T. nitidum e T. aurifrons, respectivamente (Fig. 2). Para estes últimos sítios, a ocupação predominante de uma espécie foi observada considerando todas as coletas, embora uma pequena variação nas proporções de espécies durante os meses de amostragem tenha sido verificada.

Arquitetura dos Ninhos. As espécies de Trypoxylon utilizaram ninhos-armadilha de diferentes comprimentos $(\mathrm{F}=$ $33,34 ; \mathrm{P}=0,00)$ e diâmetros $(\mathrm{F}=143,80 ; \mathrm{P}=0,00)$, mas foi observada alguma sobreposição (Tab. III). O teste de MannWhitney revelou que valores médios de comprimento e diâmetro do tubo são significativamente diferentes para
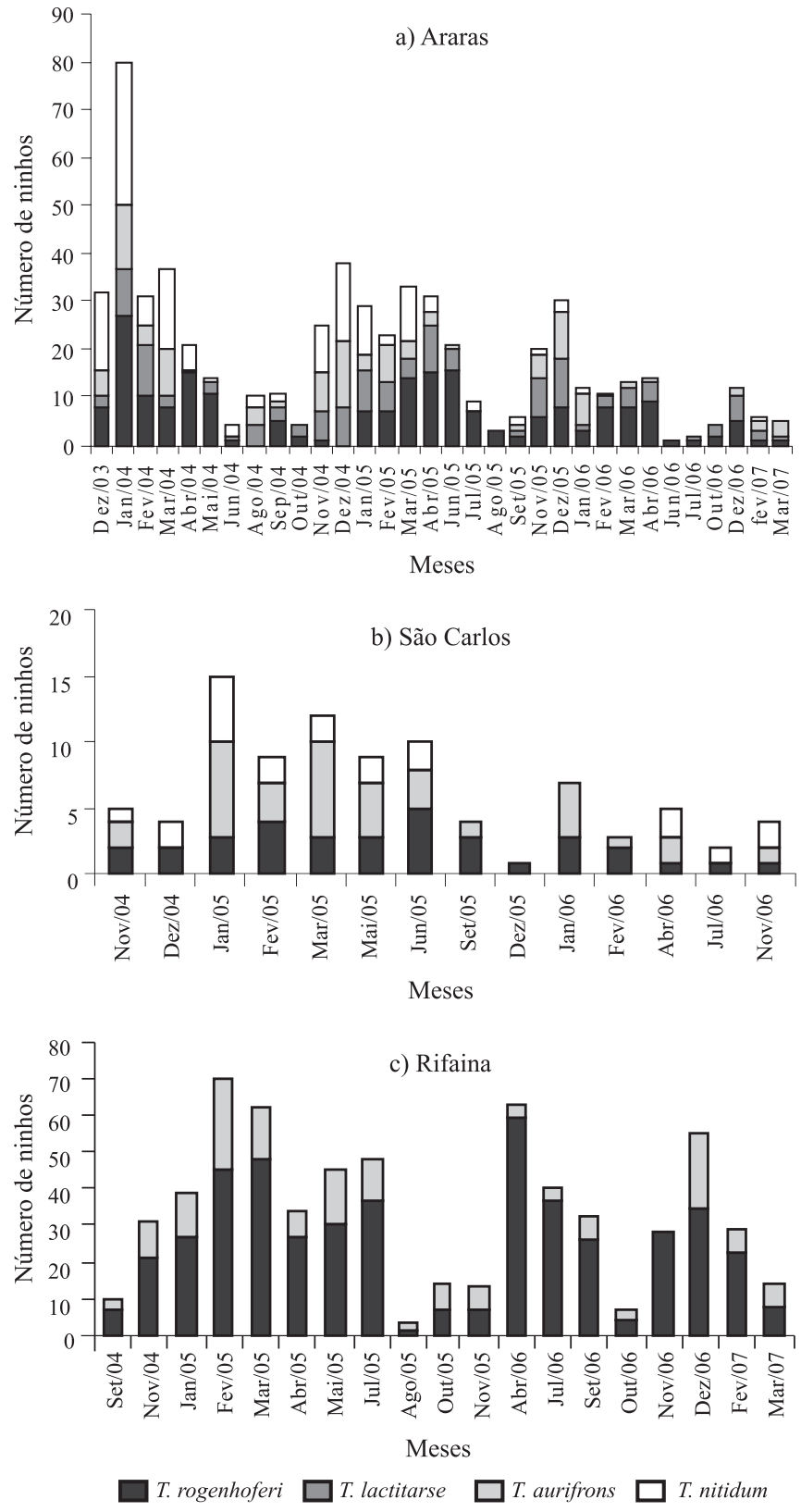

Fig. 1. Número de ninhos fundados por espécies de Trypoxylum em cada amostragem em três localidades do estado de São Paulo.

algumas comparações (Tab. III). Apenas para T. rogenhoferi os diâmetros dos tubos utilizados nas três localidades foram significativamente diferentes $(\mathrm{F}=7,45 ; \mathrm{P}=0,04)$.

Desconsiderando Trypoxylon sp. (devido ao baixo número de ninhos obtidos), a arquitetura intranidal foi semelhante para as espécies estudadas: os ninhos apresentaram parede de fundo (deposição de barro no fundo do tubo) e parede de fechamento (parede de barro construída na extremidade do tubo). As células foram construídas em séries lineares, divididas por paredes de barro (paredes de partição). Foram observadas ainda células de fundo (célula vazia no fundo do tubo) e células vestibulares (célula vazia entre a parede de fechamento e a última célula aprovisionada) (Tab. IV). 
a) Araras

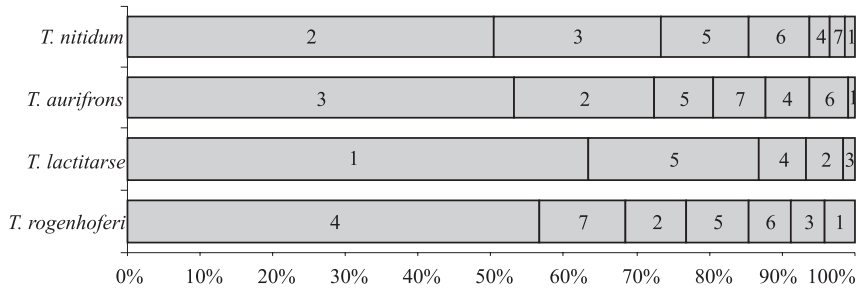

b) São Carlos

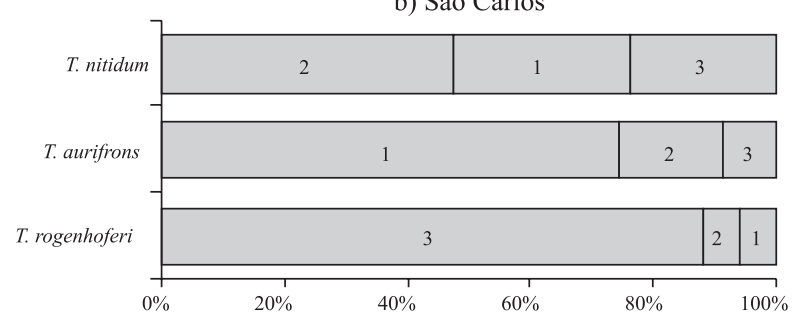

c) Rifaina

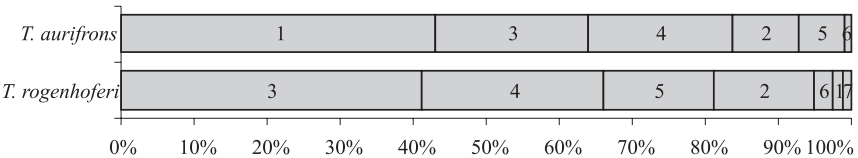

Fig. 2. Porcentagem do número de ninhos fundados por espécies de Trypoxylon em diferentes sítios de nidificação de três localidades do estado de São Paulo (cada sítio de nidificação é representado pelo número no interior da barra).

Presas coletadas. Foram amostradas 1.809 aranhas, pertencentes a 11 famílias - Araneidae $(93,6 \%)$, Tetragnathidae $(4,5 \%)$, Salticidae $(0,8 \%)$ e Anyphaenidae $(0,5 \%)$. As famílias Lycosidae, Mimetidae, Nephilidae, Oxyopidae, Philodromidae, Scytodidae e Uloboridae representaram, juntas, $0,6 \%$ das presas.

Mil e trinta aranhas foram provenientes de 38 ninhos de $T$. rogenhoferi, 12 ninhos de T. lactitarse, 15 de T. aurifrons e 15 de T. nitidum. A alta porcentagem de imaturos amostrados (779 aranhas) dificultou a identificação das espécies, pois os indivíduos nesta fase não apresentam as estruturas da genitália definitivamente formadas. As aranhas das famílias Mimetidae, Nephilidae, Scytodidae e Uloboridae foram coletadas em ninhos de Trypoxylon que não apresentaram emergência de adultos e, consequentemente, não foi possível associá-las às espécies de Trypoxylon que as utilizaram como presas.

As espécies analisadas utilizaram aranhas de diferentes gêneros e/ou espécies (Tab. V). Nas três localidades, $T$. rogenhoferi apresentou preferência por aranhas do gênero Alpaida. T. lactitarse, uma espécie mais generalista, capturou principalmente Eustala sp. T. aurifrons e T. nitidum capturaram preferencialmente Eustala fuscovittata e Eustala sp., respectivamente.

Mortalidade e inimigos naturais. Foram amostradas 4.431 células de T. rogenhoferi, 826 de T. lactitarse, 1.252 de $T$. aurifrons e 887 de T. nitidum. A maior taxa de mortalidade ocorreu no estágio de pupa para as quatro espécies (Tab. VI) e foi devida, principalmente, a causas não determinadas.
Melittobia (Hymenoptera, Eulophidae) foi o principal parasitóide das espécies de Trypoxylon. Além deste, vespas das famílias Ichneumonidae, Chalcididae e Chrysididae e moscas da família Sarcophagidae também foram observadas (Tab. VI).

Como esperado, as taxas de emergência e parasitismo mantiveram-se constantes ao longo das coletas em Araras e Rifaina em relação ao número de células produzidas pelas espécies estudadas.

\section{DISCUSSÃO}

A maior diversidade dos ninhos-armadilha oferecida justifica a maior taxa de ocupação verificada neste trabalho quando comparada à de outros estudos (Loyola \& Martins 2006; Aguiar \& Garófalo 2004).

Muitos fatores podem afetar as taxas de nidificação por espécies de himenópteros solitários que utilizam cavidades preexistentes, como a oferta de cavidades, matéria-prima para construção e disponibilidade de recursos para aprovisionamento, entre outros (Roubik 1989). Cada uma das áreas está particularmente estruturada de acordo com a disponibilidade destes fatores e estas diferenças contribuem para justificar as diferenças na composição local de espécies. A maior riqueza de espécies em Araras se deve a especificidades do ambiente local e não apenas à disponibilidade de cavidades durante um maior período de amostragem, pois a oferta excessiva de ninhos-armadilha e o tempo de amostragem nas outras áreas não devem ter sido limitantes para que outras espécies nidificassem nestes sítios; por outro lado, a maior abundância verificada em Rifaina pode ser reflexo de uma comunidade estruturada há mais tempo.

Nossos achados confirmam a influência da sazonalidade, possivelmente associada à temperatura média mensal, sobre a biologia de nidificação das espécies neotropicais e demonstram a ocorrência temporalmente sincrônica destas espécies, principalmente na estação quente e úmida. Taxas elevadas de nidificação na estação quente e chuvosa foram igualmente relatadas nos estudos realizados por Camillo et al. (1995), Assis \& Camillo (1997) e Loyola \& Martins (2006) em T. rogenhoferi (Camillo et al. 1994) e T. lactitarse (Camillo et al. 1993; Buschini et al. 2006) e T. opacum (Buschini \& Wolff 2006).

A não reposição dos indivíduos emergidos aos seus sítios de origem explica a diminuição do número de fundações ao longo do período amostrado, embora os sítios permanecessem disponíveis para novas fundações. As novas fundações foram

Tabela I. Número de ninhos fundados por espécies de Trypoxylon em três localidades do estado de São Paulo.

\begin{tabular}{ccccccc}
\hline Localidades & $T$. & $T$. & $T$. & $T$. & $T$. & Total \\
& rogenhoferi & lactitarse & aurifrons & nitidum & sp & \\
\hline Araras & 212 & 120 & 118 & 141 & 12 & 603 \\
Rifaina & 478 & & 151 & & & 629 \\
São Carlos & 35 & & 34 & 21 & & 90 \\
Total & 725 & 120 & 303 & 162 & 12 & 1322 \\
\hline
\end{tabular}


Tabela II. Correlação (r) entre o número de ninhos fundados pelas espécies de Trypoxylon e a temperatura média mensal ou a precipitação total no período de coletas.

\begin{tabular}{lcccc}
\hline & T. rogenhoferi & T. lactitarse & T. aurifrons & T. nitidum \\
\hline Temperatura & $0,42^{*}$ & $0,48^{*}$ & $0,43^{*}$ & $0,49^{*}$ \\
Precipitação & 0,10 & 0,06 & 0,11 & 0,21 \\
\hline * $\mathrm{P}<0,05$ & & &
\end{tabular}

produzidas por fêmeas e machos da mesma espécie, mantendo a ocupação sítio-preferencial observada. Análises genéticas do material estocado utilizando marcadores poderosos (genes mitocondriais e microssatélites) permitirão evidenciar se os novos ninhos foram fundados por fêmeas remanescentes ou se houve entrada de material genético novo ao longo do tempo.

As espécies de Trypoxylon estudadas demonstraram preferência pelo diâmetro da cavidade de nidificação, confirmando dados de outros autores (Assis \& Camillo 1997; Budrine et al. 2004; Buschini et al. 2006, Buschini \& Wolff 2006; Coville 1982, Coville \& Coville 1980; Garcia \& Adis 1995). Estes estudos demonstraram que o diâmetro escolhido oscila entre um limite inferior, determinado pelo tamanho do corpo da vespa e da presa utilizada (Garcia \& Adis 1995) e um limite superior, definido pela espessura das paredes de barro, visto que paredes muito finas não conferem proteção contra parasitóides (Coville \& Coville 1980).

As espécies estudadas aprovisionaram seus ninhos principalmente com aranhas da família Araneidae; no entanto, elas diferiram quanto às espécies utilizadas (Coville 1982). Trabalhos prévios em $T$. rogenhoferi e T. lactitarse relataram igual preferência pelos gêneros e espécies de aranhas verificados neste trabalho (Buschini et al. 2006; Camillo \& Brescovit 1999, 2000; Camillo et al. 1994; Garcia \& Adis 1995). O forrageamento espécie-específico também foi observado em T. tenoctitlan (Coville \& Coville 1980) e T. antropovi (Camillo 1999).

Embora tenha sido descrito que as espécies de aranhas capturadas por uma determinada espécie possam variar entre as áreas estudadas e as épocas de nidificação (Camillo \& Brescovit 1999), estudos conduzidos em diferentes áreas e épocas relataram que estas vespas apresentam preferência relativamente constante pelas espécies de presas forrageadas (Buschini \& Wolff 2006; Buschini et al. 2006; Camillo 1999, Camillo et al. 1994; Garcia \& Adis 1995).

O parasitismo nas espécies de Trypoxylon causou cerca de $23 \%$ das mortes, porém não foi a principal causa de mortalidade, como observado em outras espécies de

Tabela III. Comprimento $(\mathrm{cm})$ e diâmetro $(\mathrm{mm})$ dos ninhos-armadilha utilizados pelas espécies de Trypoxylon. Teste de Mann-Whitney (U); * $\mathrm{P}>0.05$. Letras iguais indicam médias semelhantes.

\begin{tabular}{ccccccc}
\hline & \multicolumn{3}{c}{ Comprimento } & \multicolumn{3}{c}{ Diâmetro } \\
\cline { 2 - 7 } & Min & $\operatorname{Max}$ & $\bar{X} \pm S D$ & Min & Max & $\bar{X} \pm S D$ \\
\hline T. rogenhoferi & 10,5 & 61,5 & $27,8 \pm 8,5^{\text {a }}$ & 5,1 & 19,2 & $9,0 \pm 2,0^{\mathrm{d}}$ \\
T. lactitarse & 8,6 & 44,0 & $21,8 \pm 5,8^{\mathrm{b}}$ & 4,5 & 17,5 & $9,2 \pm 2,3^{\mathrm{d}}$ \\
T. aurifrons & 11,7 & 46,7 & $24,0 \pm 6,6^{\mathrm{c}}$ & 3,1 & 16,6 & $6,7 \pm 1,7^{\mathrm{e}}$ \\
T. nitidum & 14,4 & 46,7 & $24,3 \pm 5,4^{\mathrm{c}}$ & 3,5 & 11,4 & $6,5 \pm 1,6^{\mathrm{e}}$ \\
\hline
\end{tabular}

Tabela IV. Arquitetura dos ninhos fundados por espécies de Trypoxylon em ninhos-armadilha em três localidades do estado de São Paulo (A = amplitude de variação; $\mathrm{N}=$ número de observações).

\begin{tabular}{lcccc}
\hline $\begin{array}{l}\text { Estrutura } \\
\text { do ninho }\end{array}$ & $\begin{array}{c}T . \\
\text { rogenhoferi lactitarse }\end{array}$ & $\begin{array}{c}T . \\
\text { aurifrons }\end{array}$ & $\begin{array}{c}T . \\
\text { nitidum }\end{array}$ \\
\hline $\begin{array}{l}\text { Ninhos com mais de } \\
\text { uma parede de fundo }\end{array}$ & 3 & - & 2 & - \\
$\begin{array}{l}\text { Ninhos com mais de } \\
\text { Ninho }\end{array}$ & 55 & 2 & 7 & 10
\end{tabular}

uma parede de

fechamento

Número de células $\quad \mathrm{A}=1-17 \quad \mathrm{~A}=4-15 \quad \mathrm{~A}=1-12 \quad \mathrm{~A}=2-13$

por ninho (ninhos $\quad 7 \pm 3 \quad 8 \pm 2 \quad 5 \pm 2 \quad 6 \pm 2$

completos) $\quad \mathrm{N}=471 \quad \mathrm{~N}=79 \quad \mathrm{~N}=215 \quad \mathrm{~N}=87$

Comprimento do $\quad \mathrm{A}=7-36 \quad \mathrm{~A}=7-14,2 \quad \mathrm{~A}=5-21,2 \quad \mathrm{~A}=1-24,9$

ninho $(\mathrm{cm}) \quad 18,6 \pm 8 \quad 10,1 \pm 3 \quad 12,4 \pm 4 \quad 6,3 \pm 7$

$\begin{array}{ccccc}\text { Comprimento da } & \mathrm{N}=37 & \mathrm{~N}=4 & \mathrm{~N}=28 & \mathrm{~N}=25 \\ & -0,5-4,0 & - & \mathrm{A}=0,6-4,0 & -\end{array}$

célula de fundo $(\mathrm{cm}) \quad 1,9 \pm 1 \quad 1,2 \pm 1$

$\mathrm{N}=82 \quad \mathrm{~N}=2$

Comprimento da $\mathrm{A}=0,7-5,0 \quad \mathrm{~A}=1,0-2,8 \quad \mathrm{~A}=1,0-6,5 \quad \mathrm{~A}=0,8-3,3$

célula vestibular $(\mathrm{cm}) \quad 1,5 \pm 1 \quad 1,7 \pm 1 \quad 2,2 \pm 1 \quad 1,6 \pm 1$

$\mathrm{N}=287 \quad \mathrm{~N}=268 \quad \mathrm{~N}=35 \quad \mathrm{~N}=12$

Comprimento da $\quad \mathrm{A}=1,2-2,6 \quad \mathrm{~A}=1,4-2,5 \quad \mathrm{~A}=0,7-3,3 \quad \mathrm{~A}=0,9-2,5$

célula de macho $(\mathrm{cm}) \quad 1,7 \pm 0.4 \quad 1,8 \pm 0.3 \quad 1,6 \pm 0.5 \quad 1,6 \pm 0.5$

$\mathrm{N}=56 \quad \mathrm{~N}=19 \quad \mathrm{~N}=92 \quad \mathrm{~N}=34$

Comprimento da $\mathrm{A}=1,2-2,5 \quad \mathrm{~A}=1,4-2,5 \quad \mathrm{~A}=1,0-4,8 \quad \mathrm{~A}=0,8-3,2$

célula de fêmea $(\mathrm{cm}) \quad 1,7 \pm 0.3 \quad 1,5 \pm 0.3 \quad 1,9 \pm 0.7 \quad 1,4 \pm 0.5$

$\mathrm{N}=67 \quad \mathrm{~N}=7 \quad \mathrm{~N}=86 \quad \mathrm{~N}=46$

Trypoxylon (Camillo et al. 1993; Camillo et al. 1994; Camillo \& Brescovit 1999; Garcia \& Adis 1995).

Como o principal inimigo natural das espécies aqui estudadas, a emergência de Melittobia foi descrita também em outros estudos realizados em T. rogenhoferi (Camillo et al. 1994; Loyola \& Martins 2006), T. lactitarse (Camillo et al. 1993; Loyola \& Martins 2006), T. politum (Molumby 1995) e $T$. antropovi (Camillo 1999).

Adultos de Ichneumonidae emergiram dos ninhos amostrados, como observado em ninhos de T. lactitarse (Assis \& Camillo 1997; Buschini et al. 2006), T. rogenhoferi (Assis \& Camillo 1997; Camillo et al. 1994), T. tenoctitlan (Coville \& Coville 1980) e T. opacum (Buschini \& Wolff 2006). Adultos da família Chrysididae também emergiram de ninhos de T. tenoctitlan (Coville \& Coville 1980), T. lactitarse (Assis \& Camillo 1997; Buschini et al. 2006; Camillo et al. 1993), T. rogenhoferi (Assis \& Camillo 1997; Camillo et al. 1994; Garcia \& Adis 1995), T. opacum (Buschini \& Wolff 2006) e T. aestivale (Camillo 1999). Buschini et al. (2006) e Buschini \& Wolff (2006) verificaram que moscas da família Sarcophagidae emergiram de ninhos de T. lactitarse e T. opacum, respectivamente. Os nossos dados e os da literatura indicam, portanto, que as espécies de Trypoxylon analisadas neste trabalho têm inimigos naturais comuns.

Ocupação diferencial do habitat. A nidificação pelas espécies de Trypoxylon apresentou um padrão sítiopreferencial, caracterizado pela presença predominante de uma espécie em um sítio específico. Para as quatro espécies, esta 
Tabela V. Número de aranhas amostradas em ninhos fundados por espécies de Trypoxylon em três localidades do estado de São Paulo: a = Araras, $r$ = Rifaina e $\mathrm{s}=$ São Carlos.

\begin{tabular}{|c|c|c|c|c|}
\hline \multirow[t]{2}{*}{ Classificação } & $T$. & $T$. & $T$. & \multirow{2}{*}{$\begin{array}{c}T . \\
\text { nitidum }\end{array}$} \\
\hline & \multicolumn{3}{|c|}{ rogenhoferi lactitarse aurifrons } & \\
\hline Araneidae & $182^{\mathrm{a}}, 373^{\mathrm{r}}, 44^{\mathrm{s}}$ & 99 & $85^{\mathrm{a}}, 35^{\mathrm{r}}, 30^{\mathrm{s}}$ & $112^{\mathrm{a}}, 49$ \\
\hline Acacesia $\mathrm{sp}$ & $1^{\mathrm{a}}$ & & $1^{\mathrm{r}}$ & \\
\hline Alpaida sp1 & $1^{\mathrm{r}}$ & & & $4^{\mathrm{a}}$ \\
\hline Alpaida sp2 & $1^{\mathrm{r}}$ & & & \\
\hline Alpaida spp & $76^{\mathrm{a}}, 79^{\mathrm{r}}, 43^{\mathrm{s}}$ & & & \\
\hline Alpaida veniliae $^{1}$ & $42^{\mathrm{a}}, 20^{\mathrm{r}}$ & 7 & & \\
\hline Araneus spp & & 1 & $1^{\mathrm{a}}, 3^{\mathrm{r}}$ & \\
\hline Argiope argentata ${ }^{2}$ & & & $3^{\mathrm{r}}, 1^{\mathrm{s}}$ & \\
\hline Eustala spp & $3^{\mathrm{a}}$ & 12 & $3^{\mathrm{a}}$ & $11^{\mathrm{a}}, 3^{\mathrm{s}}$ \\
\hline Eustala $\mathrm{sp} 1$ & & & & $1^{\mathrm{a}}$ \\
\hline Eustala $\mathrm{sp} 2$ & & & & $1^{\mathrm{a}}$ \\
\hline $\begin{array}{r}\text { Eustala fuscovittata } \\
\text { Eustala gr. }\end{array}$ & $1^{\mathrm{r}}$ & & & \\
\hline fuscovittata & $1^{\mathrm{a}}$ & & $19^{\mathrm{a}}$ & \\
\hline Larinia $\mathrm{sp}$ & $9^{s}$ & & & \\
\hline Mecynogea $\mathrm{sp}$ & & & $1^{\mathrm{r}}$ & \\
\hline Metazygia sp & $2^{\mathrm{a}}$ & & $4^{\mathrm{a}}$ & $2^{\mathrm{a}}$ \\
\hline Ocrepeira sp & $5^{\mathrm{a}}, 10^{\mathrm{r}}, 1^{\mathrm{s}}$ & & & \\
\hline Wagneriana $\mathrm{sp}$ & & & & $1^{\mathrm{a}}$ \\
\hline $\begin{array}{l}\text { Anyphaenidae } \\
\qquad \text { Anyphaenoides }\end{array}$ & & 1 & & $2^{\mathrm{s}}$ \\
\hline clavipes $^{4}$ & & 1 & & \\
\hline Osoriella tahela ${ }^{5}$ & & & & $1^{\mathrm{s}}$ \\
\hline Umuara sp & & & & $1^{\mathrm{s}}$ \\
\hline Lycosidae & & 1 & & \\
\hline Oxyopidae & & & & $1^{\mathrm{s}}$ \\
\hline Philodromidae & & & & $2^{\mathrm{s}}$ \\
\hline Paracleocnemis sp & & & & $1^{\mathrm{s}}$ \\
\hline Salticidae & & & & $1^{\mathrm{a}}, 4^{\mathrm{s}}$ \\
\hline Lyssomanes $\mathrm{sp}$ & & & & $2^{\mathrm{s}}$ \\
\hline Tetragnathidae & $1^{\mathrm{a}}$ & 7 & $1^{\mathrm{s}}$ & \\
\hline Leucauge sp & & & $1^{\mathrm{s}}$ & \\
\hline Tetragnatha & $1^{\mathrm{a}}$ & & & \\
\hline
\end{tabular}

predominância foi observada nas diferentes coletas. Em Araras, T. aurifrons e T. nitidum apresentaram uma ligeira variação na preferência pelos sítios de nidificação ao longo das coletas e tal resultado pode ser devido à grande proximidade destes sítios $(50 \mathrm{~m})$.

A preferência por sítios de nidificação ganha relevância ao se considerar que, nas três áreas de estudo, os sítios escolhidos são próximos entre si e, aparentemente, apresentam níveis de temperatura, luminosidade e umidade relativa semelhantes. Em dois dos sítios de nidificação em Araras, foi observada uma baixa taxa de nidificação pelas espécies de Trypoxylon. Um destes sítios diferenciava-se dos demais por estar em edificação baixa, mais à sombra e nas proximidades de um curso de água. Nos três anos de coleta realizados em Araras, este sítio foi ocupado quase exclusivamente por Podium denticulatum F. Smith, 1856 (Sphecidae) (dados não publicados). O segundo sítio referido se caracterizava por ser uma construção aberta, onde foram capturadas espécies de eumeníneos. Vale ressaltar que estas espécies apresentam inimigos comuns com as espécies de Trypoxylon aqui analisadas (Assis \& Camillo 1997).

O padrão de distribuição dos animais tem sido interpretado principalmente como (1) o sub-produto de diferenças fisiológicas, morfológicas e bionômicas, (2) o resultado de diferentes histórias evolutivas ou (3) o resultado de competição interespecífica, seguida de subseqüente especialização para um habitat ótimo (Storch \& Frynta 2000).

As alternativas (1) e (2) parecem não ser relevantes para explicar a ocupação diferencial do habitat pelas espécies de Trypoxylon, dada a proximidade filogenética das mesmas. Alternativamente, a competição interespecífica pode ser uma explicação para a segregação espacial verificada.

A teoria da competição prediz que a sobreposição de exigências entre espécies em um habitat deve ser minimizada pela segregação temporal ou espacial das mesmas. Dessa forma, preferências por diferentes habitats são comumente explicadas como resultado da especialização ao habitat em que o sucesso reprodutivo de uma espécie não é reduzido pela competição com outras espécies (Begon et al. 2006).

A coexistência temporal destas espécies de Trypoxylon que parecem apresentar demandas similares sugere que a partição do habitat pode ser interpretada como resultado de competição interespecífica, seguida de posterior especialização para habitats ótimos (Rosenzweig 1981), como demonstrado em comunidades de himenópteros por Dietrich \& Wehner (2002).

No entanto, as espécies estudadas aparentemente não apresentam sobreposição de recursos para nidificação e forrageamento, visto que utilizam ninhos-armadilhas de diferentes dimensões e aprovisionam seus ninhos com diferentes espécies de aranhas. Esta suposição é corroborada por Camillo \& Brescovit (2000), que reportaram baixa sobreposição de nichos entre T. rogenhoferi $\mathrm{e}$ T. lactitarse em razão destas espécies não competirem em termos de aprovisionamento.

A ocorrência de 'competição aparente' pode ser uma explicação alternativa para esta ocupação diferencial do habitat. Holt (1977) propôs este termo para indicar a redução da densidade populacional de uma espécie quando a densidade de uma segunda espécie aumenta, sendo esta interação mediada pelo aumento numérico de uma terceira espécie de um nível trófico superior. De acordo com este autor, o compartilhamento de inimigos naturais, como observado nas espécies de Trypoxylon, pode estruturar uma comunidade da mesma forma que a competição por recursos o faz. Como resultado, espécies que compartilham inimigos naturais devem evoluir para ocupar em simpatria diferentes áreas livres destes inimigos (Jeffries \& Lawton 1984).

Padrões de distribuição em mosaico têm sido largamente documentados em comunidades de insetos herbívoros (Rott \& Godfray 2000). Morris et al. $(2001,2004)$ e van Veen et al. (2005) postulam que, assim como neste grupo, a partição do habitat observada em outras comunidades de insetos pode ser explicada por competição aparente devida ao compartilhamento de parasitóides. 
Tabela VI. Número de células, taxas de emergência e mortalidade em espécies de Trypoxylon de três localidades do estado de São Paulo. o = ovo, 1 = larva, $\mathrm{p}=$ pupa.

\begin{tabular}{|c|c|c|c|c|}
\hline Localidade & $\begin{array}{c}T . \\
\text { rogenhoferi }\end{array}$ & $\begin{array}{c}T . \\
\text { lactitarse }\end{array}$ & $\begin{array}{c}T . \\
\text { aurifrons }\end{array}$ & $\begin{array}{c}T . \\
\text { nitidum }\end{array}$ \\
\hline \multicolumn{5}{|l|}{ Araras } \\
\hline Células & 1481 & 848 & 602 & 803 \\
\hline Emergência & $831(56 \%)$ & $475(56 \%)$ & $264(44 \%)$ & $505(63 \%)$ \\
\hline Mortalidade & $\begin{array}{c}114^{\mathrm{o}}, 21^{1} \\
515^{\mathrm{p}}\end{array}$ & $\begin{array}{c}106^{\circ}, 6^{1} \\
261^{\mathrm{p}}\end{array}$ & $\begin{array}{c}36^{\circ}, 10^{1}, \\
292^{\mathrm{p}}\end{array}$ & $\begin{array}{c}51^{\circ}, 24^{1}, \\
223^{p}\end{array}$ \\
\hline Parasitóide & $207(14 \%)$ & $143(17 \%)$ & $128(21 \%)$ & $129(16 \%)$ \\
\hline Melittobia & 192 & 100 & 125 & 113 \\
\hline Ichneumonidae & 9 & 40 & 1 & 9 \\
\hline Chrysididae & 1 & 3 & 2 & 7 \\
\hline Sarcophagidae & 5 & 0 & 0 & 0 \\
\hline $\begin{array}{l}\text { Causas } \\
\text { desconhecidas } \\
\text { Rifaina }\end{array}$ & $443(30 \%)$ & $230(27 \%)$ & $210(35 \%)$ & $169(21 \%)$ \\
\hline Células & 3007 & & 583 & \\
\hline Emergência & $1975(66 \%)$ & & $351(60 \%)$ & \\
\hline Mortalidade & $\begin{array}{c}260^{\circ}, 80^{1}, \\
692^{\mathrm{p}}\end{array}$ & & $\begin{array}{c}34^{\mathrm{o}}, 8^{1}, \\
190^{\mathrm{p}}\end{array}$ & \\
\hline Parasitóide & $255(8 \%)$ & & $80(14 \%)$ & \\
\hline Melittobia & 218 & & 61 & \\
\hline Ichneumonidae & 10 & & 1 & \\
\hline Sarcophagidae & 25 & & 6 & \\
\hline Chrysididae & 0 & & 12 & \\
\hline Chalcididae & 0 & & 0 & \\
\hline Formicidae & 2 & & 0 & \\
\hline $\begin{array}{r}\text { Causas } \\
\text { desconhecidas } \\
\text { São Carlos }\end{array}$ & $777(26 \%)$ & & $152(26 \%)$ & \\
\hline Células & 185 & & 142 & 84 \\
\hline Emergência & $100(54 \%)$ & & $81(57 \%)$ & $37(44 \%)$ \\
\hline Mortalidade & $24^{\circ}, 2^{1}, 59^{\mathrm{p}}$ & & $17^{\circ}, 1^{1}, 43^{p}$ & $11^{\mathrm{o}}, 36^{\mathrm{p}}$ \\
\hline Parasitóide & $28(15 \%)$ & & $17(12 \%)$ & $12(14 \%)$ \\
\hline Melittobia & 26 & & 15 & 11 \\
\hline Ichneumonidae & 2 & & & \\
\hline Chrysididae & & & 2 & 1 \\
\hline $\begin{array}{r}\text { Causas } \\
\text { desconhecidas }\end{array}$ & $57(31 \%)$ & & $44(31 \%)$ & $35(42 \%)$ \\
\hline
\end{tabular}

Se a competição aparente é uma alternativa aceitável para a nidificação em mosaico das espécies de Trypoxylon, o reconhecimento dos sítios mais vantajosos e a partição do habitat por estas espécies pode ser resultado de aquisições de dicas cognitivas do habitat (Storch \& Frynta 2000), dentre as quais: (i) a presença dos primeiros colonizadores de cada espécie, demonstrando a qualidade do sítio, como observado por Mönkkönen et al (1997,1999); (ii) a ocorrência de parasitismo em sítio já colonizado por uma espécie, servindo como dica para que outra espécie evite a divisão dos custos do parasitismo.

Neste trabalho, dados sobre a biologia de nidificação de quatro espécies de Trypoxylon em três localidades do estado de São Paulo foram relatados. Embora apresentem muitos aspectos semelhantes da biologia de nidificação, as espécies parecem não competir por importantes recursos (substrato para nidificação e presas). Alternativamente, a ocupação diferencial do habitat aparentemente homogêneo pelas espécies de Trypoxylon foi justificada pela competição aparente mediada pelos inimigos naturais comuns.

A demonstração de que a competição aparente produz padrões de dominância de diferentes espécies em diferentes sítios de nidificação, como sugerida pelos nossos dados, é relevante do ponto de vista teórico e prático. As propostas de manejo de espécies usualmente sugerem o enriquecimento de habitats com elementos que possam ser objeto de competição ou que, de alguma forma, limitem o crescimento das populaçõesalvo. Entretanto, se este enriquecimento potencializar o estabelecimento da competição aparente e segregar as espécies em sítios específicos do habitat, o risco de extinções locais pode aumentar, tendo em vista a fragmentação crescente do habitat.

Dadas a abundância e a capacidade de nidificação das espécies neotropicais de Trypoxylon em ninhos-armadilha, este grupo de vespas permite testar se a competição aparente gera partição do habitat e verificar os efeitos desta partição nas taxas de extinção local, contribuindo para o conhecimento dos fatores que devem ser considerados ao se delinear estratégias conservacionistas.

Agradecimentos. Ao Dr. Adhemar Rodrigues Alves, dono da Fazenda Rio Branco (Rifaina - SP), ao Dr. Sérvio Túlio Pires Amarante e José Carlos Serrano pela identificação das espécies de Trypoxylon, a Rogério Oliveira Souza pela ajuda nas coletas e à Dra. Maria José de Oliveira Campos pela leitura do manuscrito e sugestões. Às agências financiadoras CNPQ (ADB) e FAPESP.

\section{REFERÊNCIAS}

Aguiar, C. M. L. \& C. A. Garófalo. 2004. Nesting biology of Centris (Hemisiella) tarsata (Hymenoptera, Apidae, Centridini). Revista Brasileira de Zoologia 21: 477-486.

Assis, J. M. F. \& E. Camillo. 1997. Diversidade, sazonalidade e aspectos biológicos de vespas solitárias (Hymenoptera: Sphecidae: Vespidae) em ninhos armadilhas na região de Ituiutaba, MG. Anais da Sociedade Entomológica do Brasil 26: 335-347.

Ayres, M.; M. Ayres Jr.; D. L. Ayres \& A. S. Santos. 2005. BioEstat 4.0: Aplicações Estatísticas nas Áreas das Ciências Biológicas e Médicas. Belém, Sociedade Civil Mamirauá, xii + $324 \mathrm{p}$.

Begon, M.; J. L. Harper \& C. R. Townsend. 2006. Ecology: individuals, populations and communities. ( $2^{\text {nd }}$ Ed.). Oxford. Blackwell. xii +738 p.

Brockmann, H. J. \& A. Grafen. 1992. Sex ratios and life-history patterns of a solitary wasp, Trypoxylon (Trypargilum) politum (Hymenoptera: Sphecidae). Behavioral Ecology and Sociobiology 30: 7-27.

Budriene, A.; E. Budrys \& Z. Nevonitre. 2004. Solitary Hymenoptera Aculeata inhabiting trap-nests in Lithuania: nesting cavity choice and niche overlap. Latvijas Entomologs 41: 19-31.

Buschini, M. L. T. \& L. L. Wolff. 2006. Notes on the biology of Trypoxylon (Trypargilum) opacum Brèthes (Hymenoptera; Crabronidae) in Southern Brazil. Brazilian Journal of Biology 66: $915-926$.

Buschini, M. L. T.; L. L. Wolff \& F. Niesing. 2006. Nesting biology of Trypoxylon (Trypargilum) lactitarse (Hymenoptera; Crabronidae) in trap-nests in southern Brazil. Brazilian Journal of Biology 66: $161-171$.

Camillo, E. 1999. Bionomics of Trypoxylon (Trypargilum) antropovi 
and Trypoxylon (Trypargilum) aestivale (Hymenoptera: Sphecidae). Iheringia 87: 29-36.

Camillo, E. \& A. D. Brescovit. 1999. Aspectos biológicos de Trypoxylon (Trypargilum) lactitarse Saussure e Trypoxylon (Trypargilum) rogenhoferi Kohl (Hymenoptera: Sphecidae) em ninhos-armadilha, com especial referência a suas presas. Anais da Sociedade Entomológica do Brasil 28: 251-262.

Camillo, E. \& A. D. Brescovit. 2000. Spider prey (Araneae) of Trypoxylon (Trypargilum) rogenhoferi (Hymenoptera: Sphecidae) in southeastern Brazil. Revista de Biologia Tropical 48: 647656.

Camillo, E.; C. A. Garófalo \& J. C. Serrano. 1994. Observações sobre a biologia de Trypoxylon (Trypargilum) rogenhoferi Kohl (Hymenoptera: Sphecidae). Anais da Sociedade Entomológica do Brasil 23: 299-310.

Camillo, E.; C. A. Garófalo; G. Muccillo \& J. C. Serrano. 1993. Biological observation on Trypoxylon (Trypargilum) lactitarse Saussure in Southeastern Brazil (Hymenoptera, Sphecidae). Revista Brasileira de Entomologia 37: 769-778.

Camillo, E.; C. A. Garófalo; Serrano, J. C. \& G. Muccillo. 1995. Diversidade e abundância sazonal de abelhas e vespas solitárias em ninhos-armadilha (Hymenoptera, Apocrita, Aculeata). Revista Brasileira de Entomologia 39: 459-470.

Coville, R. E. 1982. Wasps of the genus Trypoxylon subgenus Trypargilum in North America. Berkeley. University of California Press. v+147 p.

Coville, R. E. \& P. L. Coville. 1980. Nesting biology and male behavior of Trypoxylon (Trypargilum) tenoctitlan in Costa Rica (Hymenoptera: Sphecidae). Annals of the Entomological Society of America 73: 110-119.

Dietrich, B. \& R. Wehner. 2003. Sympatry and allopatry in two desert ant sister species: how do Cataglyphis bicolor and C. savignyi coexist? Oecologia 136: 63-72.

Garcia, M. V. B. \& J. Adis. 1995. Comportamento de nidificação de Trypoxylon (Trypargilum) rogenhoferi Kohl (Hymenoptera, Sphecidae) em uma floresta inundável de várzea na Amazônia Central. Amazoniana 13: 259-282.

Gauld, I. D. \& B. Bolton (Eds.). 1996. The Hymenoptera. (2 $2^{\text {nd }}$ Ed.). New York. Oxford University Press. xi+332 p.

Hanson, P. E. \& A. S. Menke. 1996. The Sphecid Wasps (Sphecidae), p. 621-649. In: P. E. Hanson \& I. D. Gauld (eds.). The Hymenoptera of Costa Rica. New York. Oxford University Press. Xiii+920 p.

Holt, R. D. 1977. Community modules, p. 333-349. In: A. C. Gange \& V. K. Brown (eds.). Multitrophic interactions in terrestrial systems. Oxford. Blackwell Science. $x+446$ p.

Jeffries M. J. \& J. H. Lawton. 1984. Enemy free space and the structure of ecological communities. Biological Journal of the Linnean Society 23: 269-286.

Loyola, R. D. \& R. P. Martins. 2006. Trap-nest occupation by solitary wasps and bees (Hymenoptera: Aculeata) in a forest urban remanent Neotropical Entomology 35: 41-48.

Molumby, A. 1995. Dynamics of parasitism in the organ-pipe wasp, Trypoxylon politum: effects of spatial scale on parasitoid functional response. Ecological Entomology 20: 159-168.

Mönkkönen M.; P. Helle; G. J. Niemi \& K. Montgomery. 1997. Heterospecific attraction affects community structure and migrant abundances in northern breeding bird communities. Canadian Journal of Zoology 75: 2077-2083.

Mönkkönen M.; R. Härdling; J. T. Forsman \& J. Tuomi. 1999. Evolution of heterospecific attraction: using other species as cues in habitat selection. Evolutionary Ecology 13: 91-104.

Morato, E. F. \& R. P. Martins. 2006. An overview of proximate factors affecting the nesting behavior of solitary wasps and bees (Hymenoptera: Aculeata) in preexisting cavities in wood. Neotropical Entomology 35: 285-298.

Morris, R. J.; C. B. Müller \& H. C. J. Godfray. 2001. Field experiments testing for apparent competition between primary parasitoids mediated by secondary parasitoids. Journal of Animal Ecology 70: $301-309$.

Morris, R. J.; O. T. Lewis \& H. C. J. Godfray. 2004. Experimental evidence for apparent competition in a tropical forest food web. Nature 428: $310-313$

O'Neill, K. M. 2001. Solitary Wasps: Behavior and Natural History. Ithaca. Cornell University Press. Xii+406 p.

Peruquetti, R. C. \& M. A. Del Lama. 2003. Alocação sexual e seleção sexo-dependente para tamanho de corpo em Trypoxylon rogenhoferi Kohl (Hymenoptera, Sphecidae). Revista Brasileira de Entomologia 47: 581-588.

Rosenzweig, M. L. 1981. A theory of habitat selection. Ecology 62 327-335.

Rott, A. S. \& H. C. J. Godfray. 2000. The structure of a leafminerparasitoid community. Journal of Animal Ecology 69: 274289.

Roubik, D. W. 1989. Ecology and Natural History of Tropical Bees. Cambridge. Cambridge University Press. $x+514$ p.

Santoni, M. S. \& M. A. Del Lama. 2007. Nesting biology of the trapnesting Neotropical wasp Trypoxylon (Trypargilum) aurifrons Shuckard (Hymenoptera, Crabronidae). Revista Brasileira de Entomologia 51: 369-376.

Storch D. \& D. Frynta. 2000. Evolution of habitat selection: stochastic acquisition of cognitive clues? Evolutionary Ecology 13: 591600 .

van Veen, F. J. F.; P. D. van Holland \& H. C. J. Godfray. 2005. Stable coexistence in experimental insect communities due to densitymediated and trait-mediated indirect effects. Annual Review of Entomology 51: 187-208

Zar, J. H. 1999. Biostatistical Analysis. (4 ${ }^{\text {th }}$ Ed.). Upper Saddle. Prentice Hall. xi+929 p 